\title{
An improved PR controller current tracking control strategy research for Active Power Filter
}

\author{
Mingcai Pan, Ziqiang Xi , Miao Zhou \\ Hubei Collaborative Innovation Center for High-efficient Utilization of Solar Energy, Hubei \\ University of Technology, Wuhan, Hubei, China
}

panmcai@foxmail.com,Xizq820@163.com,zhoum2014@163.com

Keywords: Current tracking; PR controller; Pre-corrected Tustin transformation

Abstract: The current tracking control is the important part of APF (Active Power Filter) system. Since APF compensation current contain high harmonic components, the traditional Tustin discretization method distort the current in high frequency, which makes the resonant frequency of the PR controller shifted and affects the compensation effect of APF. In this paper, pre-corrected Tustin transformation method is used to improve traditional PR controller, which can track sinusoidal signal of specified frequency with no steady-state error and compensate the harmonics of specified frequency selectively.

\section{Introduction}

Compensation current tracking control technique affect the harmonic compensation effect of APF directly, which usually transform the voltage and current in the three-phase static coordinate system to rotational $\mathrm{d}-\mathrm{q}$ coordinate system with angular velocity of each order inharmonic through coordinate transformation. Then a simple PI controller is used to realize the current floating control, achieving active and reactive power decoupling control at the same time. But the converter is controlled by PI controller, the output voltage has a phase and amplitude difference. According to the characteristics of infinite gain for a particular frequency sinusoidal signal of the resonant controller, this paper proposes further improvements to the traditional discretization method while adopting PR controller to control the output characteristics. Thus the harmonic suppression effect of Active Power Filter is improved.

\section{The current loop controlin static coordinate system based on the PR controller}

The synchronous rotating reference coordinate system current loop control structure can achieve high tracking precision in the corresponding harmonic frequency. But designation of multiple independent PI controller and a large number of matrix calculation are needed. The controlled variable of current loop control turn into time-varying AC signals in static coordinate system, thus the corresponding improvement to the controller is needed in the system. It can be achieved by transform the controller transfer function in $d-q-n$ coordinate to $\alpha-\beta$ coordinate system. The process is given as follows:

$$
G_{P I n}^{\alpha \beta}(s)=\frac{1}{2}\left(G_{P I n}^{d q n}(s+j n \omega)+G_{P I n}^{d q n}(s-j n \omega)\right)
$$

Where $n$ is the order of harmonic, $\omega$ is the fundamental angular frequency, $G_{P I n}^{d q n}$ is the transfer function of PI controlin d-q-n coordinate system, namely: 


$$
G_{P I n}^{d q n}=K_{i P n}+\frac{K_{i I n}}{s}
$$

(2) is added to (1):

$$
G_{P I n}^{\alpha \beta}(s)=K_{P n}+\frac{K_{R n} s}{s^{2}+(n \omega)^{2}}
$$

Specified order of harmonic controller in $\alpha-\beta$ coordinate system shows as (3), which is also known as proportional resonant controller, namely the PR controller. The second item on the right of (3) has the property of the improper integral, which has infinite gains at the frequency of $n \omega$ and low gains at other frequencies. Then it can implement a zero steady-state error tracking with sinusoidal signal of the same frequency and adjust the control of compensating the specified order of harmonic. Thus lots of coordinate transformation can be reduced by using the PR controller in $\alpha-\beta$ coordinate system And no steady-state error tracking to high frequency sinusoidal signals without high control gains is realized.

In order to improve the stability of the control performance and digital implementation, the resonance frequency of the PR controller should be as same as the harmonic frequency of the grid as shown in (3). When the grid changes transiently, the controller still keeps high gain control characteristics of specified order of harmonics and also has the robust frequency stabilization at the same time. With the transfer function of controller becomes: $\quad \omega_{n}=n \omega$

$$
G_{P I n}^{\alpha \beta}(s)=K_{P n}+\frac{2 K_{R n} \omega_{c} s}{s^{2}+2 \omega_{c} s+\omega_{n}^{2}}
$$

Although the gain at the resonant frequency in (4) is limited, the system has superior steady-state error characteristics, which is different to (3). The proportional parameter $\omega_{c}$ will affect the stability of the control system. The bandwidth of the resonance frequency control system can be changed by $K_{P n}$, which also help to improve the robustness of the controller on the system frequency change.

\section{The discretization of PR controller}

The PR controller is implemented by using DSP, which discretize the transform function in s-domain. When Tustin transformation is used, then

$$
s=\frac{2}{T} \cdot \frac{z-1}{z+1}
$$

The bilinear transformation is one to one mapping, which can avoid frequency aliasing and ensure the discrete frequency characteristic. But this method still distort in high frequency.

The purpose of the modified bilinear transformation is to select the key frequency $\omega_{\mathrm{r}}$. In order to realize the desired goals, should translate before Tustin transformation, since

to

$$
D\left(s / \omega_{1}\right) \quad D\left(s / \omega_{A}\right)
$$

$$
D\left(s / \omega_{A}\right)=D\left(\frac{s}{\omega_{1}} \frac{\omega_{1}}{\omega_{A}}\right)
$$


It equals to add a scale factor $\frac{\omega_{1}}{\omega_{A}}=\frac{\omega_{1}}{2 / T \tan \omega_{1} T / 2}$ to the original system transfer function. Then the transfer function of the fixed bilinear transformation turns into:

$$
D(z)=\left.D(s)\right|_{s=\frac{\omega_{1}}{\tan \left(\omega_{1} T / 2\right)} \frac{z-1}{z+1}}
$$

Due to the frequency fixing, the continuous frequency characteristic of $\omega_{1}$ is same to discrete frequency characteristic, which still distort at other frequencies. Thus the selected harmonic can be compensated.

The conventional amplitude phase frequency characteristics curve of PR controller as is shown in figure 1 according to (3), taking the fifth harmonic frequency as resonance frequency for example. It obtains the highest control gain when the frequency is $250 \mathrm{HZ}$. When compensating the selected harmonic $(5,7,9,11$ and 13 level cases), the amplitude phase frequency characteristics curve of the improved PR the controller as is shown in figure 2.

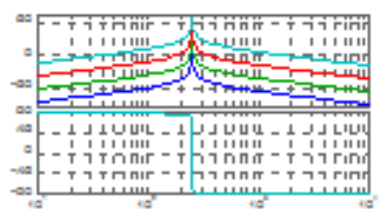

Figure1. Bode diagrams of resonant controller with Tustin transformation

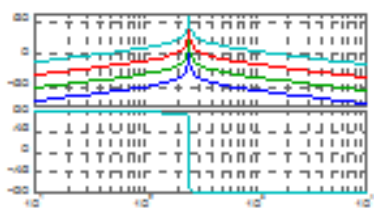

Figure2. Bode diagrams of resonant controller with fixed Tustin transformation

\section{Results}

The compensation current control algorithm of the improve PR controller has been verified by computer simulation and experimental research. The simulation and experimental parameters of the algorithm mare as follows: (1) Power supply:380V/50Hz;(2)Load: rectifier bridge with RL load, $\mathrm{R}=1.5 \Omega, \mathrm{L}=1.13 \mathrm{mH}$; (3)Input inductance of the power line: $100 \mu \mathrm{H}$; (4) Output inductance of the Active Power Filter:1.35mH; (5)DC-link voltage: 750V.

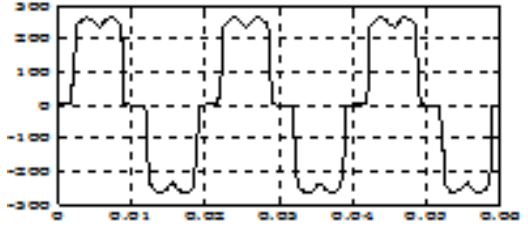

Figure3. Load side of the current simulation waveforms

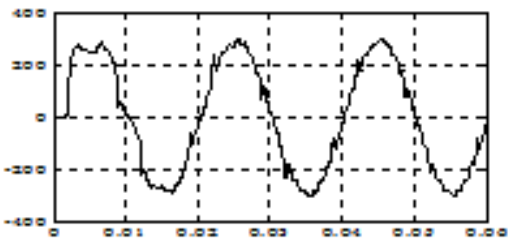

Figure4.Compensated source side current simulation waveforms 


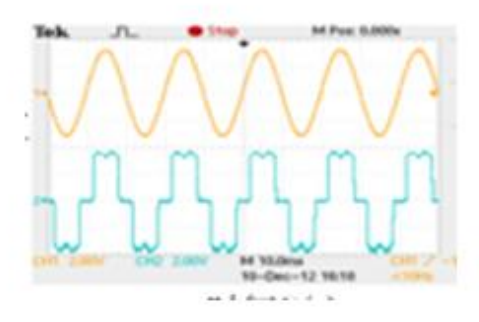

Figure5. Load side of the current test waveforms

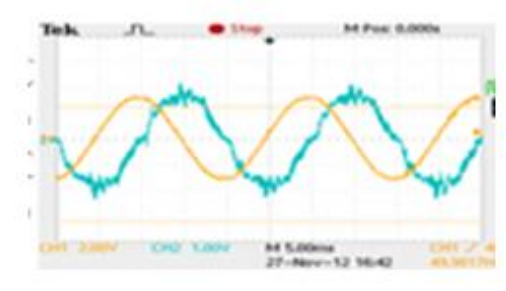

Figure6.Compensated source side current measured waveforms

\section{Summary}

The improved PR controller adopts the fixed frequency to ensure the continuous frequency characteristic of $\omega_{1}$ same to discrete frequency characteristic, but still distorts at other frequencies. Thus the selected harmonic can be compensated. Simulation and experimental results show that there search of improved PR controller current tracking control strategy for APF has a superior performance.

\section{References}

[1]Yang Y,Zhou K,Blaabjerg F. Harmonics suppression for single-phase grid-connected PV systems in different operation modes[C] .Applied Power Electronics Conference and Exposition:APEC,2013 Twenty-Eighth Annual IEEE,IEEE,2013:889-896.

[2]Yu Jingrong, Su Mei, Sun Yao. Improved repetitive control and its optimization for active power filter [J].Transaction of China Electro technical society, 2012, 27(2):235-242.

[3]Singh M, Khadkikar, V, Chandra A, et al. Grid interconnection of renewable energy sources at the distribution level with power-quality improvement features [J]. IEEE Transactions on Power Delivery, 2011 , Page(s): 307 - 315

[4]Zhou Juan, Zhang Yong, Geng Yiwen, et al. An improved proportional resonant control strategy in the static coordinate for four-leg active power filters [J]. Proceedings of the CSEE, 2012, 32(6): 111-120.

[5]Tumbelaka H H, Miyatake M. Simple integration of three-phase shunt active power filter and photovoltaic generation system with Fibonacci search based MPPT[C]. 2010 IEEE Symposium on Industrial Electronics \& Applications (ISIEA), 2010: 94-99. 